\title{
A Completeness Theorem for Kleene Algebras and the Algebra of Regular Events*
}

\author{
Dexter Kozen \\ Department of Computer Science \\ Cornell University \\ Ithaca, New York 14853 \\ kozen@cs.cornell.edu
}

\begin{abstract}
We give a finitary axiomatization of the algebra of regular events involving only equations and equational implications. Unlike Salomaa's axiomatizations, the axiomatization given here is sound for all interpretations over Kleene algebras.
\end{abstract}

\section{Introduction}

Kleene algebras are algebraic structures with operators $+, \cdot{ }^{*}, 0$, and 1 satisfying certain axioms. They arise in various guises in a number of settings: relational algebra [22, 23], semantics and logics of programs [14, 24], automata and formal language theory $[18,19]$, and the design and analysis of algorithms [1, 21, 12].

An important example of a Kleene algebra is $\mathbf{R e g}_{\Sigma}$, the family of regular sets over a finite alphabet $\Sigma$. The equational theory of this structure has been called the algebra of regular events. This theory was first studied by

* Infor. and Comput. 110:2 (May 1994), 366-390. A preliminary version of this paper appeared as [16]. 
Kleene [13], who posed axiomatization as an open problem. Salomaa [28] gave two complete axiomatizations of the algebra of regular events in 1966 , but these axiomatizations depended on rules of inference that are not sound in general under nonstandard interpretations. Redko [25] proved in 1964 that no finite set of equational axioms could characterize the algebra of regular events. The algebra of regular events and its axiomatization is the subject of the extensive monograph of Conway [8]. The bulk of Conway's treatment is infinitary. In 1981, we gave a complete infinitary equational deductive system for the algebra of regular events that is sound over all *-continuous Kleene algebras [14]. A completeness theorem for relational algebras with *, a proper subclass of Kleene algebras, was given by $\mathrm{Ng}$ and Tarski [23, 22], but their axiomatization relies on the presence of a converse operator. Schematic equational axiomatizations for the algebra of regular events, necessarily representing infinitely many equations, have been given by Krob [17] and Bloom $[6]$.

There is some disagreement regarding the definition of Kleene algebras [8, 24, 14]. The literature contains several inequivalent definitions. In this paper we introduce yet another: a Kleene algebra is any model of the equations and equational implications given in $\S 2$.

By general considerations of equational logic, the axioms of Kleene algebra listed in $\S 2$, along with the usual axioms for equality, instantiation, and rules for the introduction and elimination of implications, constitute a complete deductive system for the universal Horn theory of Kleene algebras (the set of universally quantified equational implications

$$
\alpha_{1}=\beta_{1} \wedge \cdots \wedge \alpha_{n}=\beta_{n} \rightarrow \alpha=\beta
$$

true in all Kleene algebras) [30].

The main result of this paper is that this deductive system is complete for the algebra of regular events. In other words, two regular expressions $\alpha$ and $\beta$ over $\Sigma$ denote the same regular set in $\boldsymbol{R e g}_{\Sigma}$ if and only if the equation $\alpha=\beta$ is a logical consequence of the axioms. Equivalently, $\boldsymbol{R e g}_{\Sigma}$ is the free Kleene algebra on free generators $\Sigma$.

This gives a more satisfactory solution to Kleene's question than Salomaa's solution, since the axiomatization is sound over an entire array of important nonstandard interpretations arising in computer science. The result is proved by encoding the classical combinatorial constructions of the theory of finite automata, e.g. state minimization, algebraically. 
There is an extensive literature on the algebra of regular events $[8,4,17]$ and much of the development of this paper is a recapitulation of previous work. For example, the construction of a transition matrix representing a finite automaton equivalent to a given regular expression is essentially implicit in the work of Kleene [13] and appears in Conway's monograph [8]; the algebraic approach to the elimination of $\epsilon$-transitions appears in the work of Kuich and Salomaa [19] and Sakarovitch [27]; and the results on the closure of Kleene algebras under the formation of matrices essentially go back to Conway's monograph [8] and the thesis of Backhouse [4]. We extend this program by showing how to encode algebraically two fundamental constructions in the theory of finite automata:

- determinization of an automaton via the subset construction, and

- state minimization via equivalence modulo a Myhill-Nerode equivalence relation.

We then use the uniqueness of the minimal deterministic finite automaton to obtain completeness.

Conway states a similar theorem without proof in the latter part of his book [8, Theorem 5, p. 108]. Krob [17], based on work of Boffa [7], and Archangelsky [3] have recently independently obtained similar results based on different techniques.

\subsection{Examples of Kleene Algebras}

Kleene algebras abound in computer science. We have already mentioned the regular sets $\boldsymbol{R e g}_{\Sigma}$.

In the area of relational algebra, the family of binary relations on a set with the operations of $\cup$ for + , relational composition

$$
R \cdot S=\{(x, z) \mid \exists y(x, y) \in R,(y, z) \in S\}
$$

for $\cdot$, the empty relation for 0 , the identity relation for 1 , and reflexive transitive closure for ${ }^{*}$ constitute a Kleene algebra.

In semantics and logics of programs, Kleene algebras are used to model programs in Dynamic Logic and Dynamic Algebra [14, 24].

In the design and analysis of algorithms, $n \times n$ Boolean matrices and matrices over the so-called min, + algebra are used to derive efficient algorithms for reachability and shortest paths in directed graphs [1, 21]. A Kleene algebra in which + gives the vector sum of two polygons and $\cdot$ gives the convex 
hull of the union of two polygons has been used to solve a cycle problem in graphs [12]. These Kleene algebras appear in [1, 21, 12] in the guise of closed semirings, which are similar to the $\mathbf{S}$-algebras of Conway [8] (also called Kleene semirings). Closed semirings and $\mathbf{S}$-algebras are defined in terms of an infinitary summation operator $\sum$, whose sole purpose, it seems, is to define ${ }^{*}$. These structures are all closely related; the precise relationship is drawn in [15].

\subsection{Salomaa's Axiomatizations}

Before one can fully appreciate the axiomatization of $\S 2$, it is important to understand Salomaa's axiomatizations [28] and their limitations. Let $R_{\Sigma}$ denote the interpretation of regular expressions over $\Sigma$ in the Kleene algebra $\boldsymbol{R e g}_{\Sigma}$ in which

$$
R_{\Sigma}(a)=\{a\}, a \in \Sigma
$$

This is called the standard interpretation.

Salomaa [28] presented two axiomatizations $F_{1}$ and $F_{2}$ for the algebra of regular events and proved their completeness. Aanderaa [2] independently presented a system similar to Salomaa's $F_{1}$. Backhouse [4] gave an algebraic

version of $F_{1}$. These systems are equational except for one rule of inference in each case that is sound under the standard interpretation $R_{\Sigma}$, but not sound in general over other interpretations.

To describe the system $F_{1}$, let us say a regular expression possesses the empty word property (EWP) if the regular set it denotes under $R_{\Sigma}$ contains the null string $\epsilon$. The EWP can be characterized syntactically: a regular expression $\alpha$ has the EWP if either

- $\alpha=1$;

- $\alpha=\beta^{*}$ for some $\beta$;

- $\alpha$ is a sum of regular expressions, at least one of which has the EWP; or

- $\alpha$ is a product of regular expressions, both of which have the EWP. The system $F_{1}$ contains the rule

$$
\frac{\gamma+\alpha \beta=\beta, \quad \alpha \text { does not have the EWP }}{\alpha^{*} \gamma=\beta} .
$$


The rule (2) is sound under the standard interpretation $R_{\Sigma}$, but the proviso " $\alpha$ does not have the EWP" is not algebraic in the sense that it is not preserved under substititution. Consequently, (2) is not valid under nonstandard interpretations. For example, if $\alpha, \beta$, and $\gamma$ are the single letters $a, b$ and $c$ respectively, then (2) holds; but it does not hold after the substitution

$$
a \mapsto 1 \quad b \mapsto 1 \quad c \mapsto 0 .
$$

Another way to say this is that (2) must not be interpreted as a universal Horn formula. Salomaa's system $F_{2}$ is somewhat different from $F_{1}$ but suffers from a similar drawback.

In contrast, the axioms for Kleene algebra given in $\S 2$ below are all equations or equational implications in which the symbols are regarded as universally quantified, so substitution is allowed.

\section{Axioms for Kleene Algebra}

A Kleene algebra is an algebraic structure

$$
\mathcal{K}=\left(K,+, \cdot{ }^{*}, 0,1\right)
$$

satisfying the following equations and equational implications:

$$
\begin{aligned}
a+(b+c) & =(a+b)+c \\
a+b & =b+a \\
a+0 & =a \\
a+a & =a \\
a(b c) & =(a b) c \\
1 a & =a \\
a 1 & =a \\
a(b+c) & =a b+a c \\
(a+b) c & =a c+b c \\
0 a & =0 \\
a 0 & =0 \\
1+a a^{*} & \leq a^{*} \\
1+a^{*} a & \leq a^{*}
\end{aligned}
$$




$$
\begin{aligned}
& b+a x \leq x \rightarrow a^{*} b \leq x \\
& b+x a \leq x \rightarrow b a^{*} \leq x
\end{aligned}
$$

where $\leq$ refers to the natural partial order on $\mathcal{K}:$

$$
a \leq b \quad \leftrightarrow \quad a+b=b
$$

Instead of (16) and (17), we might take the equivalent axioms

$$
\begin{aligned}
& a x \leq x \rightarrow a^{*} x \leq x \\
& x a \leq x \rightarrow x a^{*} \leq x .
\end{aligned}
$$

Axioms (3-6) say that $(K,+, 0)$ is an idempotent commutative monoid. Axioms $(7-9)$ say that $(K, \cdot, 1)$ is a monoid. Axioms $(3-13)$ say that $(K,+, \cdot 0,1)$ is an idempotent semiring.

The remaining axioms (14-19) deal with *. They say essentially that

* behaves like the Kleene star operator of formal language theory or the reflexive transitive closure operator of relational algebra. Using (14) and the distributivity axiom (11), we see that

$$
b+a a^{*} b \leq a^{*} b,
$$

thus the left hand side of the implication (16) is satisfied when $a^{*} b$ is substituted for $x$; moreover, (16) says that $a^{*} b$ is the least element of $\mathcal{K}$ for which this is true. In short, $a^{*} b$ is the least prefixpoint of the monotone map $x \mapsto b+a x$.

Axioms (16-19) are studied by Pratt [24], who attributes (16) and (17) to Schröder and Dedekind. The equivalence of (16) and (18) (and, by symmetry, of (17) and (19)) are proved in [24].

No attempt has been made to reduce the axioms above to a minimal set and no claim is made as to their independence.

All the structures mentioned in $§ 1$, in particular $\mathbf{R e g}_{\Sigma}$, are Kleene algebras.

\subsection{Elementary consequences}

In this section we derive some basic consequences of the Kleene algebra axioms. These properties are quite elementary and have been observed before 
by many different authors. We do not claim any of them as original results, but include them merely for the sake of completeness. We refer the reader to $[8,4]$ for a comprehensive introduction.

It is straightforward to verify that the relation $\leq$ is a partial order, and is monotone with respect to all the Kleene algebra operators in the sense that if $a \leq b$, then $a c \leq b c, c a \leq c b, a+c \leq b+c$, and $a^{*} \leq b^{*}$. With respect to $\leq, \mathcal{K}$ is an upper semilattice with join given by + and minimum element 0 .

Basic properties of ${ }^{*}$ such as

$$
\begin{aligned}
1 & \leq a^{*} \\
a & \leq a^{*} \\
a^{*} a^{*} & =a^{*} \\
a^{* *} & =a^{*}
\end{aligned}
$$

are also easily derived. See [8] for formal proofs.

Lemma 1 In any Kleene algebra, $a^{*}$ is the unique element satisfying (14) and (16). It is also the unique element satisfying (15) and (17).

Proof. By (14), $a^{*}$ satisfies the inequality

$$
1+a x \leq x
$$

when substituted for $x$. By (16), it is the least such element. Thus $a^{*}$ is unique.

The second assertion is proved by a symmetric argument involving (15) and (17).

Proposition 2 In any Kleene algebra, the inequalities (14) and (15) can be strengthened to equations:

$$
\begin{aligned}
& 1+a a^{*}=a^{*} \\
& 1+a^{*} a=a^{*} .
\end{aligned}
$$

Proof. The inequality $1+a a^{*} \leq a^{*}$ is given by (14). To show

$$
a^{*} \leq 1+a a^{*},
$$


it suffices by (16) and (9) to show that

$$
1+a\left(1+a a^{*}\right) \leq 1+a a^{*} .
$$

But this is immediate from (14) and the monotonicity of - and + .

The proof of $1+a^{*} a=a^{*}$ is symmetric.

The following result was observed by Pratt [24].

Proposition 3 Under the assumptions (3-14), the implications (16) and (18) are equivalent. Under the assumptions (3-13) and (15), the implications (17) and (19) are equivalent.

Proof. We prove the first statement; the second is symmetric. First assume (16) and the premise of (18). By assumption, $a x \leq x$, therefore $x+a x \leq x$. By (16), $a^{*} x \leq x$. Discharging the hypothesis, we obtain the implication (18).

Now assume (18) and the premise of (16). By assumption, $b+a x \leq x$, thus $b \leq x$ and $a x \leq x$. By (18), $a^{*} x \leq x$, and by monotonicity, $a^{*} b \leq a^{*} x$, therefore $a^{*} b \leq x$. Discharging the hypothesis, we obtain the implication (16).

The following proposition is a key tool in the completeness proof of $\S 5$.

\section{Proposition 4 In all Kleene algebras,}

$$
a x=x b \rightarrow a^{*} x=x b^{*} .
$$

Proof. Suppose first that $a x \leq x b$. Then

$$
a x b^{*} \leq x b b^{*}
$$

by monotonicity, and

$$
x+x b b^{*} \leq x b^{*}
$$

by (14) and distributivity, therefore by monotonicity,

$$
\begin{aligned}
x+a x b^{*} & \leq x+x b b^{*} \\
& \leq x b^{*} .
\end{aligned}
$$


By (16),

$$
a^{*} x \leq x b^{*} .
$$

By a symmetric argument using (15) and (17),

$$
x b \leq a x \rightarrow x b^{*} \leq a^{*} x .
$$

The proposition follows from these two implications.

Corollary 5 In all Kleene algebras,

$$
(c d)^{*} c=c(d c)^{*} .
$$

Proof. Substitute $c$ for $x, c d$ for $a$, and $d c$ for $b$ in Proposition 4.

Corollary 6 Let $p$ be an invertible element of a Kleene algebra with inverse $p^{-1}$. Then

$$
p^{-1} a^{*} p=\left(p^{-1} a p\right)^{*} .
$$

Proof. We have

$$
\begin{aligned}
a^{*} p & =\left(p p^{-1} a\right)^{*} p \\
& =p\left(p^{-1} a p\right)^{*}
\end{aligned}
$$

by Corollary 5 . The result follows by multiplying on the left by $p^{-1}$.

Proposition 7 In all Kleene algebras,

$$
(a+b)^{*}=a^{*}\left(b a^{*}\right)^{*} .
$$

Proof. To show

$$
(a+b)^{*} \leq a^{*}\left(b a^{*}\right)^{*},
$$

observe that

$$
\begin{aligned}
1 & \leq a^{*}\left(b a^{*}\right)^{*} \\
a a^{*}\left(b a^{*}\right)^{*} & \leq a^{*}\left(b a^{*}\right)^{*} \\
b a^{*}\left(b a^{*}\right)^{*} & \leq\left(b a^{*}\right)^{*} \\
& \leq a^{*}\left(b a^{*}\right)^{*}
\end{aligned}
$$


therefore

$$
\begin{aligned}
1+(a+b) a^{*}\left(b a^{*}\right)^{*} & \leq 1+a a^{*}\left(b a^{*}\right)^{*}+b a^{*}\left(b a^{*}\right)^{*} \\
& \leq a^{*}\left(b a^{*}\right)^{*} .
\end{aligned}
$$

Then (20) follows from (16).

To show the reverse inequality, we use the monotonicity of all the operators:

$$
\begin{aligned}
a^{*}\left(b a^{*}\right)^{*} & \leq(a+b)^{*}\left((a+b)(a+b)^{*}\right)^{*} \\
& \leq(a+b)^{*}\left((a+b)^{*}\right)^{*} \\
& \leq(a+b)^{*} .
\end{aligned}
$$

\section{Matrices over a Kleene Algebra}

Under the natural definitions of the operators $+, \cdot{ }^{*}, 0$, and 1 , the family $\mathcal{M}(n, \mathcal{K})$ of $n \times n$ matrices over a Kleene algebra $\mathcal{K}$ again forms a Kleene algebra. This is a standard result proved for various classes of algebras in $[8,4]$. None of Conway's or Backhouse's algebras are Kleene algebras in our sense, and their results do not imply the result we need here, so we must provide an explicit proof. Nevertheless many of the techniques are similar.

Define + and $\cdot$ on $\mathcal{M}(n, \mathcal{K})$ to be the usual operations of matrix addition and multiplication, respectively, $Z_{n}$ the $n \times n$ zero matrix, and $I_{n}$ the $n \times n$ identity matrix. The partial order $\leq$ is defined on $\mathcal{M}(n, \mathcal{K})$ by

$$
A \leq B \quad \leftrightarrow \quad A+B=B
$$

Under these definitions, it is routine to verify

Lemma 8 The structure

$$
\left(\mathcal{M}(n, \mathcal{K}),+, \cdot, Z_{n}, I_{n}\right)
$$

is an idempotent semiring; that is, the Kleene algebra axioms (3-13) are satisfied. 
Proof. See $[8,4]$.

The definition of $E^{*}$ for $E \in \mathcal{M}(n, \mathcal{K})$ comes from $[8,19,9]$. We first consider the case $n=2$. This construction will later be applied inductively.

Let

$$
E=\left[\begin{array}{ll}
a & b \\
c & d
\end{array}\right]
$$

Let $f=a+b d^{*} c$ and define

$$
E^{*}=\left[\begin{array}{cc}
f^{*} & f^{*} b d^{*} \\
d^{*} c f^{*} & d^{*}+d^{*} c f^{*} b d^{*}
\end{array}\right] .
$$

This construction is motivated by a two-state finite automaton over the alphabet $\Sigma=\{a, b, c, d\}$ with states $\{s, t\}$ and transitions $s \stackrel{a}{\rightarrow} s, s \stackrel{b}{\rightarrow} t$, $t \stackrel{c}{\rightarrow} s, t \stackrel{d}{\rightarrow} t$. For each pair of states $u, v$, consider the set of input strings in $\Sigma^{*}$ taking state $u$ to state $v$ in this automaton. Each such set is regular and is represented by a regular expression corresponding to those derived for the matrix $E^{*}$ :

$$
\begin{aligned}
& s \rightarrow s:\left(a+b d^{*} c\right)^{*} \\
& s \rightarrow t:\left(a+b d^{*} c\right)^{*} b d^{*} \\
& t \rightarrow s: d^{*} c\left(a+b d^{*} c\right)^{*} \\
& t \rightarrow t:
\end{aligned}
$$

Lemma 9 The matrix $E^{*}$ defined in (21) satisfies the Kleene algebra axioms (14-17). That is,

$$
\begin{aligned}
& I+E E^{*} \leq E^{*} \\
& I+E^{*} E \leq E^{*}
\end{aligned}
$$

and for any $X$,

$$
\begin{aligned}
& E X \leq X \rightarrow E^{*} X \leq X \\
& X E \leq X \rightarrow X E^{*} \leq X
\end{aligned}
$$


Proof. We show (22) and (24). The arguments for (23) and (25) are symmetric.

The matrix inequality (22) reduces to the four inequalities

$$
\begin{aligned}
1+a f^{*}+b d^{*} c f^{*} & \leq f^{*} \\
a f^{*} b d^{*}+b\left(d^{*}+d^{*} c f^{*} b d^{*}\right) & \leq f^{*} b d^{*} \\
c f^{*}+d d^{*} c f^{*} & \leq d^{*} c f^{*} \\
1+c f^{*} b d^{*}+d\left(d^{*}+d^{*} c f^{*} b d^{*}\right) & \leq d^{*}+d^{*} c f^{*} b d^{*}
\end{aligned}
$$

in $\mathcal{K}$. These are equivalent to the inequalities

$$
\begin{aligned}
1+f f^{*} & \leq f^{*} \\
\left(1+f f^{*}\right) b d^{*} & \leq f^{*} b d^{*} \\
\left(1+d d^{*}\right) c f^{*} & \leq d^{*} c f^{*} \\
\left(1+d d^{*}\right)\left(1+c f^{*} b d^{*}\right) & \leq d^{*}\left(1+c f^{*} b d^{*}\right)
\end{aligned}
$$

respectively, which follow from the axioms and basic properties of $\S 2$.

We now establish (24). We show that (24) holds for $X$ an arbitrary column vector of length 2 ; then (24) for $X$ any $2 \times n$ matrix follows by applying this result to the columns of $X$ separately.

Let

$$
X=\left[\begin{array}{l}
x \\
y
\end{array}\right] \text {. }
$$

We need to show that under the assumptions

$$
\begin{aligned}
& a x+b y \leq x \\
& c x+d y \leq y
\end{aligned}
$$

we can derive

$$
\begin{aligned}
f^{*} x+f^{*} b d^{*} y & \leq x \\
d^{*} c f^{*} x+\left(d^{*}+d^{*} c f^{*} b d^{*}\right) y & \leq y .
\end{aligned}
$$

We establish (28) and (29) in a sequence of steps. With each step, we identify the premises from which the conclusion follows by one of the axioms or basic 
properties of $\S 2$.

$$
\begin{array}{rlrl}
a x & \leq x & & (26) \\
b y & \leq x & & (26) \\
c x & \leq y & (27) \\
d y & \leq y & & (27) \\
d^{*} y & \leq y & & (33),(18) \\
b d^{*} y & \leq b y & & (34), \text { monotonicity } \\
b d^{*} y & \leq x & & (31),(35) \\
b d^{*} c x & \leq b d^{*} y & & (32), \text { monotonicity } \\
b d^{*} c x & \leq x & & (36),(37) \\
f x & \leq x & & (30),(38) \\
f^{*} x & \leq x & & (39),(18) \\
f^{*} b d^{*} y & \leq f^{*} x & & (36), \text { monotonicity } \\
f^{*} b d^{*} y & \leq x & & (40),(41) \\
d^{*} c f^{*} x & \leq d^{*} c x & & (40), \text { monotonicity } \\
d^{*} c x & \leq d^{*} y & & (32), \text { monotonicity } \\
d^{*} c f^{*} x & \leq y & & (34),(43),(44) \\
d^{*} c f^{*} b d^{*} y & \leq d^{*} c f^{*} x & & (36), \text { monotonicity } \\
d^{*} c f^{*} b d^{*} y & \leq y & & (45),(46)
\end{array}
$$

The conclusion (28) now follows from (40) and (42) and (29) follows from (45), (34), and (47).

We now apply this argument inductively.

Lemma 10 Let $E \in \mathcal{M}(n, \mathcal{K})$. There is a unique matrix $E^{*} \in \mathcal{M}(n, \mathcal{K})$ satisfying the Kleene algebra axioms (14-17). That is,

$$
\begin{aligned}
& I+E E^{*} \leq E^{*} \\
& I+E^{*} E \leq E^{*}
\end{aligned}
$$

and for any $n \times m$ matrix $X$ over $\mathcal{K}$,

$$
\begin{aligned}
& E X \leq X \rightarrow E^{*} X \leq X \\
& X E \leq X \rightarrow X E^{*} \leq X .
\end{aligned}
$$


Proof. Partition $E$ into submatrices $A, B, C$, and $D$ such that $A$ and $D$ are square.

$$
E=\left[\begin{array}{l|l}
A & B \\
\hline C & D
\end{array}\right]
$$

By the induction hypothesis, $D^{*}$ exists and is unique. Let $F=A+B D^{*} C$. Again by the induction hypothesis, $F^{*}$ exists and is unique. We define

$$
E^{*}=\left[\begin{array}{c|c}
F^{*} & F^{*} B D^{*} \\
\hline D^{*} C F^{*} & D^{*}+D^{*} C F^{*} B D^{*}
\end{array}\right]
$$

and claim that $E^{*}$ satisfies (14-17). The proof is essentially identical to the proof of Lemma 9. We must check that the axioms and basic properties of $\S 2$ used in the proof of Lemma 9 still hold when the primitive symbols of regular espressions are interpreted as matrices of various dimensions, provided there is no type mismatch in the application of the operators.

The uniqueness of $E^{*}$ follows from Lemma 1.

Combining Lemmas 8 and 10, we obtain

Theorem 11 The structure

$$
\left(\mathcal{M}(n, \mathcal{K}),+, \cdot{ }^{*}, Z_{n}, I_{n}\right)
$$

is a Kleene algebra.

We remark that the inductive definition (53) of $E^{*}$ in Lemma 10 is independent of the partition of $E$ chosen in (52). This is a consequence of Lemma 1 , once we have established that the resulting structure is a Kleene algebra under some partition; $\mathrm{cf}$. [8, Theorem 4, p. 27].

In the proof of Lemma 10, we must check that the basic axioms and properties of $\S 2$ still hold when the primitive letters of regular expressions are interpreted as matrices of various shapes, possibly nonsquare, provided there is no type mismatch in the application of operators; e.g., one cannot add two matrices unless they are the same shape, one cannot form the matrix product $A B$ unless the column dimension of $A$ is the same as the row dimension of $B$, and one cannot form the matrix $A^{*}$ unless $A$ is square. In general, all the axioms and basic properties of Kleene algebra listed in $\S 2$ hold when the 
primitive letters are interpreted as possibly nonsquare matrices over a Kleene algebra, provided that there are no type conflicts in the application of the Kleene algebra operators. A quick review of the axioms and basic properties of $\S 2$ in light of this more general interpretation will suffice to convince the reader of the truth of this statement.

For example, the Kleene algebra theorem

$$
a x=x b \rightarrow a^{*} x=x b^{*}
$$

(Proposition 4) holds even when $a$ is an $m \times m$ matrix, $b$ is an $n \times n$ matrix, and $x$ is an $m \times n$ matrix.

For another example, consider the distributive law

$$
a(b+c)=a b+a c .
$$

Interpreting $a, b$, and $c$ as matrices over a Kleene algebra $\mathcal{K}$, this equation makes sense provided the shapes of $b$ and $c$ are the same and the column dimension of $a$ is the same as the row dimension of $b$ and $c$. Other than that, there are no type constraints. It is easy to verify that the distributive law holds for any matrices $a, b$ and $c$ satisfying these constraints.

For a more involved example, consider the equational implication of Proposition 4:

$$
a x=x b \rightarrow a^{*} x=x b^{*} .
$$

The type constraints say that $a$ and $b$ must be square (say $s \times s$ and $t \times t$ respectively) and that $x$ must be $s \times t$. Under this typing, all steps of the proof of Proposition 4 involve only well-typed expressions, thus the proof remains valid.

\section{Finite Automata}

Regular expressions and finite automata have traditionally been used as syntactic representations of the regular languages over an alphabet $\Sigma$. The relationship between these two formalisms forms the basis of a well-developed classical theory. Classical developments range from the more combinatorial $[20,11]$ to the more algebraic $[29,10,5,9,27]$. The approach taken in this paper must ultimately be attributed to Conway [8]. 
In this section we define the notion of an automaton over an arbitrary Kleene algebra. In subsequent sections, we will use this formalism to derive the classical results of the theory of finite automata (equivalence with regular expressions, determinization via the subset construction, elimination of $\epsilon$ transitions, and state minimization) as consequences of the axioms of $\S 2$.

In the following, although we consider regular expressions and automata as syntactic objects, as a matter of convenience we will be reasoning modulo the axioms of Kleene algebra. Officially, regular expressions will denote elements of $\mathcal{F}_{\Sigma}$, the free Kleene algebra over $\Sigma$. The Kleene algebra $\mathcal{F}_{\Sigma}$ is constructed by taking the quotient of the regular expressions modulo provable equivalence. The associated canonical map assigns to each regular expression its equivalence class in $\mathcal{F}_{\Sigma}$. Since we will be interpreting expressions only over Kleene algebras, and all interpretations factor through $\mathcal{F}_{\Sigma}$ via the canonical map, this usage is without loss of generality.

The following definition is closer to the algebraic definition used for example in $[8,5]$ than to the combinatorial definition used in $[20,11]$.

Definition 12 A finite automaton over $\mathcal{K}$ is a triple

$$
\mathcal{A}=(u, A, v),
$$

where $u, v \in\{0,1\}^{n}$ and $A \in \mathcal{M}(n, \mathcal{K})$ for some $n$.

The states are the row and column indices. The vector $u$ determines the start states and the vector $v$ determines the final states; a start state is an index $i$ for which $u(i)=1$ and a final state is one for which $v(i)=1$. The $n \times n$ matrix $A$ is called the transition matrix.

The language accepted by $\mathcal{A}$ is the element

$$
u^{T} A^{*} v \in \mathcal{K}
$$

For automata over $\mathcal{F}_{\Sigma}$, the free Kleene algebra on free generators $\Sigma$, this definition is essentially equivalent to the classical combinatorial definition of an automaton over the alphabet $\Sigma$ as found in [20,11]. A similar definition can be found in [8]. 
Example 13 Consider the two-state automaton in the sense of [20, 11] with states $\{p, q\}$, start state $p$, final state $q$, and transitions

$$
p \stackrel{a}{\rightarrow} p \quad q \stackrel{a}{\rightarrow} q \quad p \stackrel{b}{\rightarrow} q \quad q \stackrel{b}{\rightarrow} q .
$$

Classically, this automaton accepts the set of strings over $\Sigma=\{a, b\}$ containing at least one occurrence of $b$. In our formalism, this automaton is specified by the triple

$$
\left(\left[\begin{array}{l}
1 \\
0
\end{array}\right],\left[\begin{array}{cc}
a & b \\
0 & a+b
\end{array}\right],\left[\begin{array}{l}
0 \\
1
\end{array}\right]\right) .
$$

Modulo the axioms of Kleene algebra, we have

$$
\begin{aligned}
& {\left[\begin{array}{ll}
1 & 0
\end{array}\right] \cdot\left[\begin{array}{cc}
a & b \\
0 & a+b
\end{array}\right]^{*} \cdot\left[\begin{array}{l}
0 \\
1
\end{array}\right]} \\
& \quad=\left[\begin{array}{ll}
1 & 0
\end{array}\right] \cdot\left[\begin{array}{cc}
a^{*} & a^{*} b(a+b)^{*} \\
0 & (a+b)^{*}
\end{array}\right] \cdot\left[\begin{array}{l}
0 \\
1
\end{array}\right] \\
& =a^{*} b(a+b)^{*} .
\end{aligned}
$$

The language in $\mathbf{R e g}_{\Sigma}$ accepted by this automaton is the image under $R_{\Sigma}$ of the expression (54).

Definition 14 Let $\mathcal{A}=(u, A, v)$ be an automaton over $\mathcal{F}_{\Sigma}$, the free Kleene algebra on free generators $\Sigma$. The automaton $\mathcal{A}$ is said to be simple if $A$ can be expressed as a sum

$$
A=J+\sum_{a \in \Sigma} a \cdot A_{a}
$$

where $J$ and the $A_{a}$ are $0-1$ matrices. In addition, $\mathcal{A}$ is said to be $\epsilon$-free if $J$ is the zero matrix. Finally, $\mathcal{A}$ is said to be deterministic if it is simple and $\epsilon$-free, and $u$ and all rows of $A_{a}$ have exactly one 1 .

In Definition 14, $\epsilon$ refers to the null string. The matrix $A_{a}$ in (55) corresponds to the adjacency matrix of the graph consisting of edges labeled $a$ in the combinatorial model of automata $[11,20]$ or the image of $a$ under a linear representation map in the algebraic approach of $[29,5]$. An automaton is deterministic according to this definition iff it is deterministic in the sense of $[11,20]$.

The automaton of Example 13 is simple, $\epsilon$-free, and deterministic. 


\section{Completeness}

In this section we prove the completeness of the axioms of $\S 2$ for the algebra of regular events. Another way of stating this is that $\mathbf{R e g}_{\Sigma}$ is isomorphic to $\mathcal{F}_{\Sigma}$, the free Kleene algebra on free generators $\Sigma$, and the standard interpretation $R_{\Sigma}: \mathcal{F}_{\Sigma} \rightarrow \boldsymbol{R e g}_{\Sigma}$ collapses to an isomorphism of Kleene algebras.

The first lemma asserts that Kleene's representation theorem [13, 5, 9, 27] is a theorem of Kleene algebra.

Lemma 15 For every regular expression $\alpha$ over $\Sigma$ (or more accurately, its image in $\mathcal{F}_{\Sigma}$ under the canonical map), there is a simple automaton $(u, A, v)$ over $\mathcal{F}_{\Sigma}$ such that

$$
\alpha=u^{T} A^{*} v
$$

Proof. The proof is by induction on the structure of the regular expression. We essentially implement the combinatorial constructions as found for example in $[11,20]$. The ideas behind this construction are well known and can be found for example in [8].

For $a \in \Sigma$, the automaton

$$
\left(\left[\begin{array}{l}
1 \\
0
\end{array}\right],\left[\begin{array}{ll}
0 & a \\
0 & 0
\end{array}\right],\left[\begin{array}{l}
0 \\
1
\end{array}\right]\right)
$$

suffices, since

$$
\begin{aligned}
& {\left[\begin{array}{ll}
1 & 0
\end{array}\right] \cdot\left[\begin{array}{ll}
0 & a \\
0 & 0
\end{array}\right]^{*} \cdot\left[\begin{array}{l}
0 \\
1
\end{array}\right]} \\
& =\left[\begin{array}{ll}
1 & 0
\end{array}\right] \cdot\left[\begin{array}{ll}
1 & a \\
0 & 1
\end{array}\right] \cdot\left[\begin{array}{l}
0 \\
1
\end{array}\right] \\
& =a .
\end{aligned}
$$

For the expression $\alpha+\beta$, let $\mathcal{A}=(u, A, v)$ and $\mathcal{B}=(s, B, t)$ be automata such that

$$
\begin{aligned}
\alpha & =u^{T} A^{*} v \\
\beta & =s^{T} B^{*} t .
\end{aligned}
$$


Consider the automaton with transition matrix

$$
\left[\begin{array}{l|l}
A & 0 \\
\hline 0 & B
\end{array}\right]
$$

and start and final state vectors

$$
\left[\frac{u}{s}\right] \text { and }\left[\frac{v}{t}\right] \text {, }
$$

respectively. This construction corresponds to the combinatorial construction of forming the disjoint union of the two sets of states, taking the start states to be the union of the start states of $\mathcal{A}$ and $\mathcal{B}$, and the final states to be the union of the final states of $\mathcal{A}$ and $\mathcal{B}$. Then

$$
\left[\begin{array}{c|c}
A & 0 \\
\hline 0 & B
\end{array}\right]^{*}=\left[\begin{array}{c|c}
A^{*} & 0 \\
\hline 0 & B^{*}
\end{array}\right],
$$

and

$$
\begin{aligned}
& {\left[\begin{array}{l|l}
u^{T} & s^{T}
\end{array}\right] \cdot\left[\begin{array}{c|c}
A^{*} & 0 \\
\hline 0 & B^{*}
\end{array}\right] \cdot\left[\begin{array}{c}
v \\
t
\end{array}\right]} \\
& =u^{T} A^{*} v+s^{T} B^{*} t \\
& =\alpha+\beta .
\end{aligned}
$$

For the expression $\alpha \beta$, let $\mathcal{A}=(u, A, v)$ and $\mathcal{B}=(s, B, t)$ be automata such that

$$
\begin{aligned}
\alpha & =u^{T} A^{*} v \\
\beta & =s^{T} B^{*} t .
\end{aligned}
$$

Consider the automaton with transition matrix

$$
\left[\begin{array}{c|c}
A & v s^{T} \\
\hline 0 & B
\end{array}\right]
$$

and start and final state vectors

$$
\left[\begin{array}{l}
u \\
0
\end{array}\right] \text { and }\left[\frac{0}{t}\right] \text {, }
$$


respectively. This construction corresponds to the combinatorial construction of forming the disjoint union of the two sets of states, taking the start states to be the start states of $\mathcal{A}$, the final states to be the final states of $\mathcal{B}$, and connecting the final states of $\mathcal{A}$ with the start states of $\mathcal{B}$ by $\epsilon$-transitions (this is the purpose of the $v s^{T}$ in the upper right corner of the matrix). Then

$$
\left[\begin{array}{c|c}
A & v s^{T} \\
\hline 0 & B
\end{array}\right]^{*}=\left[\begin{array}{c|c}
A^{*} & A^{*} v s^{T} B^{*} \\
\hline 0 & B^{*}
\end{array}\right],
$$

and

$$
\begin{aligned}
& {\left[\begin{array}{l|l}
u^{T} & 0
\end{array}\right] \cdot\left[\begin{array}{c|c}
A^{*} & A^{*} v s^{T} B^{*} \\
\hline 0 & B^{*}
\end{array}\right] \cdot\left[\frac{0}{t}\right]} \\
& =u^{T} A^{*} v s^{T} B^{*} t \\
& =\alpha \beta .
\end{aligned}
$$

For the expression $\alpha^{*}$, let $\mathcal{A}=(u, A, v)$ be an automaton such that

$$
\alpha=u^{T} A^{*} v \text {. }
$$

We first produce an automaton equivalent to the expression $\alpha \alpha^{*}$. Consider the automaton

$$
\left(u, A+v u^{T}, v\right) .
$$

This construction corresponds to the combinatorial construction of adding $\epsilon$-transitions from the final states of $\mathcal{A}$ back to the start states. Using Propositions 7 and 5 ,

$$
\begin{aligned}
u^{T}\left(A+v u^{T}\right)^{*} v & =u^{T} A^{*}\left(v u^{T} A^{*}\right)^{*} v \\
& =u^{T} A^{*} v\left(u^{T} A^{*} v\right)^{*} \\
& =\alpha \alpha^{*} .
\end{aligned}
$$

Once we have an automaton for $\alpha \alpha^{*}$, we can get an automaton for $\alpha^{*}=$ $1+\alpha \alpha^{*}$ by the construction for + given above, using a trivial one-state automaton for 1 .

Now we get rid of $\epsilon$-transitions. This construction is also folklore and can be found for example in $[19,27]$. This construction models algebraically the combinatorial idea of computing the $\epsilon$-closure of a state; see [11, 20]. 
Lemma 16 For every simple automaton $(u, A, v)$ over $\mathcal{F}_{\Sigma}$, there is a simple $\epsilon$-free automaton $(s, B, t)$ such that

$$
u^{T} A^{*} v=s^{T} B^{*} t
$$

Proof. By Definition 14, the matrix $A$ can be written as a sum $A=J+A^{\prime}$ where $J$ is a $0-1$ matrix and $A^{\prime}$ is $\epsilon$-free. Then

$$
\begin{aligned}
u^{T} A^{*} v & =u^{T}\left(A^{\prime}+J\right)^{*} v \\
& =u^{T} J^{*}\left(A^{\prime} J^{*}\right)^{*} v
\end{aligned}
$$

by Proposition 7, so we can take

$$
\begin{aligned}
s^{T} & =u^{T} J^{*} \\
B & =A^{\prime} J^{*} \\
t & =v .
\end{aligned}
$$

Note that $J^{*}$ is 0 - 1 and therefore $B$ is $\epsilon$-free.

The following two results are algebraic analogs of the determinization of automata via the subset construction and the minimization of deterministic automata via the collapsing of equivalent states under a Myhill-Nerode equivalence relation. These results are apparently new, although the determinization result was recently given independently by Krob [17, §10.2] using different techniques.

Lemma 17 For every simple $\epsilon$-free automaton $(u, A, v)$ over $\mathcal{F}_{\Sigma}$ there is a deterministic automaton $(\widehat{u}, \widehat{A}, \widehat{v})$ over $\mathcal{F}_{\Sigma}$ such that

$$
u^{T} A^{*} v=\widehat{u}^{T} \hat{A}^{*} \hat{v} .
$$

Proof. We model the subset construction [11, 20] algebraically. Let $(u, A, v)$ be a simple $\epsilon$-free automaton with states $Q$. By Definition 14, $A$ can be expressed

$$
A=\sum_{a \in \Sigma} a \cdot A_{a}
$$

where each $A_{a}$ is a $0-1$ matrix. 
Let $\mathcal{P}(Q)$ denote the power set of $Q$. We identify elements of $\mathcal{P}(Q)$ with their characteristic vectors in $\{0,1\}^{n}$. For each $s \in \mathcal{P}(Q)$, let $e_{s}$ be the $\mathcal{P}(Q) \times 1$ vector with 1 in position $s$ and 0 elsewhere.

Let $X$ be the $\mathcal{P}(Q) \times Q$ matrix whose $s^{\text {th }}$ row is $s^{T}$; i.e.,

$$
e_{s}^{T} X=s^{T} .
$$

For each $a \in \Sigma$, let $\hat{A}_{a}$ be the $\mathcal{P}(Q) \times \mathcal{P}(Q)$ matrix whose $s^{\text {th }}$ row is $e_{s^{T} A_{a}}$; in other words,

$$
e_{s}^{T} \hat{A}_{a}=e_{s^{T} A_{a}} .
$$

Let

$$
\begin{aligned}
\hat{A} & =\sum_{a \in \Sigma} a \cdot \hat{A}_{a} \\
\hat{u} & =e_{u} \\
\hat{v} & =X v .
\end{aligned}
$$

The automaton $(\hat{u}, \hat{A}, \hat{v})$ is simple and deterministic.

The relationship between $A$ and $\hat{A}$ is expressed succinctly by the equation

$$
X A=\hat{A} X .
$$

Intuitively, this says that the actions of the two automata in the two spaces $\mathcal{K}^{Q}$ and $\mathcal{K}^{\mathcal{P}(Q)}$ commute with the projection $X$. To prove (56), observe that for any $s \in \mathcal{P}(Q)$,

$$
\begin{aligned}
e_{s}^{T} X A & =s^{T} A \\
& =\sum_{a \in \Sigma} a \cdot s^{T} A_{a} \\
& =\sum_{a \in \Sigma} a \cdot e_{s}^{T} A_{a} X \\
& =\sum_{a \in \Sigma} a \cdot e_{s}^{T} \hat{A}_{a} X \\
& =e_{s}^{T} \hat{A} X .
\end{aligned}
$$

By Proposition 4 (or rather its extension to nonsquare matrices as described in $\S 3$ ),

$$
X A^{*}=\hat{A}^{*} X .
$$


The theorem now follows:

$$
\begin{aligned}
\hat{u}^{T} \hat{A}^{*} \hat{v} & =e_{u}^{T} \hat{A}^{*} X v \\
& =e_{u}^{T} X A^{*} v \\
& =u^{T} A^{*} v
\end{aligned}
$$

Lemma 18 Let $(u, A, v)$ be a simple deterministic automaton and let $(\bar{u}, \bar{A}, \bar{v})$ be the equivalent minimal deterministic automaton obtained from the classical state minimization procedure [11, 20]. Then

$$
u^{T} A^{*} v=\bar{u}^{T} \bar{A}^{*} \bar{v} .
$$

Proof. In the combinatorial approach [11, 20], the unique minimal automaton is obtained as a quotient by a Myhill-Nerode equivalence relation after removing inaccessible states. We simulate this construction algebraically.

Let $Q$ denote the set of states of $(u, A, v)$. For $q \in Q$, let $e_{q} \in\{0,1\}^{Q}$ denote the vector with 1 in position $q$ and 0 elsewhere. For $a \in \Sigma$, let $A_{a}$ be the $0-1$ matrix as given in Definition 14 (55). Then

$$
A=\sum_{a \in \Sigma} a \cdot A_{a}
$$

For each $a \in \Sigma$ and $p \in Q$, let $\delta(p, a)$ be the unique state in $Q$ such that the $p^{\text {th }}$ row of $A_{a}$ is $e_{\delta(p, a)}^{T}$; i.e.,

$$
e_{p}^{T} A_{a}=e_{\delta(p, a)}^{T}
$$

The state $\delta(p, a)$ exists and is unique since the automaton is deterministic.

First we show how to get rid of unreachable states. A state $q$ is reachable if

$$
u^{T} A^{*} e_{q} \neq 0
$$

otherwise it is unreachable. Let $R$ be the set of reachable states and let $U=Q-R$ be the set of unreachable states. Partition $A$ into four submatrices $A_{R R}, A_{R U}, A_{U R}$, and $A_{U U}$ such that for $S, T \in\{R, U\}, A_{S T}$ is the $S \times T$ submatrix of $A$. Then $A_{R U}$ is the zero matrix, otherwise a state in $U$ would 
be reachable. Similarly, partition the vectors $u$ and $v$ into $u_{R}, u_{U}, v_{R}$ and $v_{U}$. The vector $u_{U}$ is the zero vector, otherwise a state in $U$ would be reachable. We have

$$
\begin{aligned}
u^{T} A^{*} v \\
=\left[u_{R}^{T} \mid 0\right] \cdot\left[\begin{array}{c|c}
A_{R R} & 0 \\
\hline A_{U R} & A_{U U}
\end{array}\right]^{*} \cdot\left[\frac{v_{R}}{v_{U}}\right] \\
=\left[u_{R}^{T} \mid 0\right] \cdot\left[\begin{array}{c|c}
A_{R R}^{*} & 0 \\
\hline A_{U U}^{*} A_{U R} A_{R R}^{*} & A_{U U}^{*}
\end{array}\right] \cdot\left[\begin{array}{c}
v_{R} \\
v_{U}
\end{array}\right] \\
=u_{R}^{T} A_{R R}^{*} v_{R} .
\end{aligned}
$$

Moreover, the automaton $\left(u_{R}, A_{R R}, v_{R}\right)$ is simple and deterministic, and all states are reachable.

Assume now that $(u, A, v)$ is simple and deterministic and all states are reachable. An equivalence relation $\equiv$ on $Q$ is called Myhill-Nerode if $p \equiv q$ implies

$$
\begin{aligned}
\delta(p, a) & \equiv \delta(q, a), \quad a \in \Sigma, \\
e_{p}^{T} v & =e_{q}^{T} v .
\end{aligned}
$$

(In combinatorial terms, $\equiv$ is Myhill-Nerode if it is respected by the action of the automaton under any input symbol $a \in \Sigma$, and the set of final states is a union of $\equiv$-classes.)

Let $\equiv$ be any Myhill-Nerode equivalence relation, and let

$$
\begin{aligned}
{[p] } & =\{q \in Q \mid q \equiv p\} \\
Q / \equiv & =\{[p] \mid p \in Q\} .
\end{aligned}
$$

For $[p] \in Q / \equiv$, let $e_{[p]} \in\{0,1\}^{Q / \equiv}$ denote the vector with 1 in position $[p]$ and 0 elsewhere. Let $Y$ be the $Q \times Q / \equiv$ matrix whose $[p]^{\text {th }}$ column is the characteristic vector of $[p]$; i.e.,

$$
e_{p}^{T} Y=e_{[p]}^{T} .
$$

For each $a \in \Sigma$, let $\bar{A}_{a}$ be the $Q / \equiv \times Q / \equiv$ matrix whose $[p]^{\text {th }}$ row is $e_{[\delta(p, a)]} ;$ i.e.,

$$
e_{[p]}^{T} \bar{A}_{a}=e_{[\delta(p, a)]}^{T} .
$$


The matrix $\bar{A}_{a}$ is well-defined by (57). Let

$$
\begin{aligned}
\bar{A} & =\sum_{a \in \Sigma} a \cdot \bar{A}_{a} \\
\bar{u}^{T} & =u^{T} Y .
\end{aligned}
$$

Also, let $\bar{v} \in\{0,1\}^{Q \equiv}$ be the vector such that

$$
e_{[p]}^{T} \bar{v}=e_{p}^{T} v
$$

The vector $\bar{v}$ is well-defined by (58). Note also that

$$
\begin{aligned}
e_{p}^{T} Y \bar{v} & =e_{[p]}^{T} \bar{v} \\
& =e_{p}^{T} v,
\end{aligned}
$$

therefore

$$
Y \bar{v}=v
$$

The automaton $(\bar{u}, \bar{A}, \bar{v})$ is simple and deterministic.

As in the proof of Lemma 17 , the action of $A$ and $\bar{A}$ commute with the linear projection $Y$ :

$$
A Y=Y \bar{A} .
$$

To prove (59), observe that for any $p \in Q$,

$$
\begin{aligned}
e_{p}^{T} A Y & =\sum_{a \in \Sigma} a \cdot e_{p}^{T} A_{a} Y \\
& =\sum_{a \in \Sigma} a \cdot e_{\delta(p, a)}^{T} Y \\
& =\sum_{a \in \Sigma} a \cdot e_{[\delta(p, a)]}^{T} \\
& =\sum_{a \in \Sigma} a \cdot e_{[p]}^{T} \bar{A}_{a} \\
& =\sum_{a \in \Sigma} a \cdot e_{p}^{T} Y \bar{A}_{a} \\
& =e_{p}^{T} Y \bar{A} .
\end{aligned}
$$


Now by Proposition 4,

$$
A^{*} Y=Y \bar{A}^{*},
$$

therefore

$$
\begin{aligned}
\bar{u}^{T} \bar{A}^{*} \bar{v} & =u^{T} Y \bar{A}^{*} \bar{v} \\
& =u^{T} A^{*} Y \bar{v} \\
& =u^{T} A^{*} v .
\end{aligned}
$$

Theorem 19 (Completeness) Let $\alpha$ and $\beta$ be two regular expressions over $\Sigma$ denoting the same regular set. Then $\alpha=\beta$ is a theorem of Kleene algebra.

Proof. Let $\mathcal{A}=(s, A, t)$ and $\mathcal{B}=(u, B, v)$ be minimal deterministic finite automata over $\mathcal{F}_{\Sigma}$ such that

$$
\begin{aligned}
& R_{\Sigma}(\alpha)=R_{\Sigma}\left(s^{T} A^{*} t\right) \\
& R_{\Sigma}(\beta)=R_{\Sigma}\left(u^{T} B^{*} v\right) .
\end{aligned}
$$

By Lemmas 15, 17, and 18, we have

$$
\begin{aligned}
& \alpha=s^{T} A^{*} t \\
& \beta=u^{T} B^{*} v
\end{aligned}
$$

as theorems of Kleene algebra. Since

$$
R_{\Sigma}(\alpha)=R_{\Sigma}(\beta),
$$

by the uniqueness of minimal automata, $\mathcal{A}$ and $\mathcal{B}$ are isomorphic. Let $P$ be a permutation matrix giving this isomorphism. Then

$$
\begin{aligned}
A & =P^{T} B P \\
s & =P^{T} u \\
t & =P^{T} v .
\end{aligned}
$$


Using Corollary 6, we have

$$
\begin{aligned}
\alpha & =s^{T} A^{*} t \\
& =\left(P^{T} u\right)^{T}\left(P^{T} B P\right)^{*}\left(P^{T} v\right) \\
& =u^{T} P\left(P^{T} B P\right)^{*} P^{T} v \\
& =u^{T} P P^{T} B^{*} P P^{T} v \\
& =u^{T} B^{*} v \\
& =\beta .
\end{aligned}
$$

\section{Open Problems}

An intriguing question is whether the techniques developed here can be extended to automata on infinite objects. An algebraic treatment of Safra's construction [26] might conceivably be used to establish completeness of the propositional $\mu$-calculus. Progress toward this goal has recently been made by Walukiewicz [31].

Another question is whether the axioms presented in $\S 2$ are complete for the universal Horn theory of the ${ }^{*}$-continuous Kleene algebras.

\section{Acknowledgements}

Roland Backhouse, John Horton Conway, Merrick Furst, Dana Scott, Vaughan Pratt, Ross Willard, and an anonymous referee provided valuable criticism and references. The support of the Danish Research Academy, the National Science Foundation through grant CCR-8901061, the John Simon Guggenheim Foundation, and the U.S. Army Research Office through ACSyAM, Mathematical Sciences Institute, Cornell University, contract DAAL03-91C-0027 is gratefully acknowledged.

\section{References}

[1] Alfred V. Aho, John E. Hopcroft, and Jeffrey D. Ullman. The Design and Analysis of Computer Algorithms. Addison-Wesley, 1975. 
[2] S. Anderaa. On the algebra of regular expressions. Appl. Math., pages 1-18, January 1965.

[3] K. V. Archangelsky. A new finite complete solvable quasiequational calculus for algebra of regular languages. Manuscript, Kiev State University, 1992.

[4] Roland Carl Backhouse. Closure algorithms and the star-height problem of regular languages. PhD thesis, Imperial College, 1975.

[5] Jean Berstel and Christophe Reutenauer. Rational Series and Their Languages. Springer-Verlag, 1984.

[6] Stephen L. Bloom and Zoltán Ésik. Equational axioms for regular sets. Technical Report 9101, Stevens Institute of Technology, May 1991.

[7] Maurice Boffa. Une remarque sur les systèmes complets d'identités rationnelles. Informatique théoretique et Applications/Theoretical Informatics and Applications, 24(4):419-423, 1990.

[8] John Horton Conway. Regular Algebra and Finite Machines. Chapman and Hall, 1971.

[9] S. Eilenberg. Automata, Languages, and Machines, volume A. Academic Press, 1974.

[10] F. Gécseg and I. Peák. Algebraic Theory of Automata. Akadémiai Kiadó, 1972.

[11] John E. Hopcroft and Jeffrey D. Ullman. Introduction to Automata Theory, Languages, and Computation. Addison-Wesley, 1979.

[12] Kazuo Iwano and Kenneth Steiglitz. A semiring on convex polygons and zero-sum cycle problems. SIAM J. Comput., 19(5):883-901, 1990.

[13] Stephen C. Kleene. Representation of events in nerve nets and finite automata. In Shannon and McCarthy, editors, Automata Studies, pages 3-41. Princeton University Press, 1956.

[14] Dexter Kozen. On induction vs. *-continuity. In Kozen, editor, Proc. Workshop Logics of Programs, volume 131 of Lect. Notes in Comput. Sci., pages 167-176. Springer-Verlag, 1981.

[15] Dexter Kozen. On Kleene algebras and closed semirings. In Rovan, editor, Proc. Math. Found. Comput. Sci. 1990, volume 452 of Lect. Notes in Comput. Sci., pages 26-47. Springer-Verlag, 1990. 
[16] Dexter Kozen. A completeness theorem for Kleene algebras and the algebra of regular events. In Proc. 6th Symp. Logic in Comput. Sci., pages 214-225. IEEE, July 1991.

[17] Daniel Krob. A complete system of B-rational identities. Theoretical Computer Science, 89(2):207-343, October 1991.

[18] Werner Kuich. The Kleene and Parikh theorem in complete semirings. In Ottmann, editor, Proc. 14th Colloq. Automata, Languages, and Programming, volume 267 of Lect. Notes in Comput. Sci., pages 212-225. EATCS, SpringerVerlag, 1987.

[19] Werner Kuich and Arto Salomaa. Semirings, Automata, and Languages. Springer-Verlag, 1986.

[20] Harry Lewis and Christos Papadimitriou. Elements of the Theory of Computation. Prentice-Hall, 1981.

[21] Kurt Mehlhorn. Data Structures and Algorithms 2: Graph Algorithms and NP-Completeness. EATCS Monographs on Theoretical Computer Science. Springer-Verlag, 1984.

[22] K. C. Ng. Relation Algebras with Transitive Closure. PhD thesis, University of California, Berkeley, 1984.

[23] K. C. Ng and A. Tarski. Relation algebras with transitive closure, abstract 742-02-09. Notices Amer. Math. Soc., 24:A29-A30, 1977.

[24] Vaughan Pratt. Dynamic algebras as a well-behaved fragment of relation algebras. In D. Pigozzi, editor, Proc. Conf. on Algebra and Computer Science, Lect. Notes in Comput. Sci. Springer-Verlag, June 1988.

[25] V. N. Redko. On defining relations for the algebra of regular events. Ukrain. Mat. Z., 16:120-126, 1964. In Russian.

[26] Shmuel Safra. On the complexity of $\omega$-automata. In Proc. 29th Symp. Foundations of Comput. Sci, pages 319-327. IEEE, October 1988.

[27] Jacques Sakarovitch. Kleene's Theorem revisited: a formal path from Kleene to Chomsky. In A. Kelemenova and J. Keleman, editors, Trends, Techniques, and Problems in Theoretical Computer Science, volume 281 of Lect. Notes in Computer Science, pages 39-50. Springer-Verlag, 1987. 
[28] Arto Salomaa. Two complete axiom systems for the algebra of regular events. J. Assoc. Comput. Mach., 13(1):158-169, January 1966.

[29] Arto Salomaa and Matti Soittola. Automata Theoretic Aspects of Formal Power Series. Springer-Verlag, 1978.

[30] A. Selman. Completeness of calculi for axiomatically defined classes of algebras. Algebra Universalis, 2:20-32, 1972.

[31] Igor Walukiewicz. Completeness result for the propositional $\mu$-calculus. In Proc. 8th Symp. Logic in Comput. Sci. IEEE, June 1993. 